\title{
High Power, Low Power, and Equality: Culture Beyond Individualism and Collectivism
}

\author{
Daphna Oyserman \\ University of Michigan
}

\begin{abstract}
Models of culture are operationalized as individualism and collectivism and have not given sufficient attention to other organizing axes - especially how a society handles power, dependence, and equality. Shavitt, Lalwani, Zhang, and Torelli (2006) make a significant contribution by first reminding the field of power distance (Hofstede, 1980) and then moving beyond a single factor to highlight benefits of Triandis' (1995) horizontal (valuing equality) individualism-collectivism and vertical (emphasizing hierarchy) individualism-collectivism model. But this approach makes it difficult to disentangle the effects of power and individualism or collectivism; priming procedures and experimental variations of power can counter this limitation. Moreover, current horizontal/vertical approaches do not distinguish between having and not having power, although social cognition research documented differential effects of high and low power on content of self-concept, relationality, and cognition, which suggest previously neglected cultural differences.
\end{abstract}

Rather than assume that the psychological models developed in the West are universal, cultural and cross-cultural psychologists have drawn attention to the possible limitations of models grounded in an American or Western European worldview, as well as limitations of data drawn from college-student subject pools that over-represent a young, high-status, and prosperous slice of any given society. Indeed, cultural and cross-cultural research argues that societies and social strata within societies differ in their socialization processes and therefore in content of self- concept, what makes for well-being, how individuals and groups are related to each other, and how people think. Particularized descriptions of various groups and societies made clear the multitude of ways of approaching the basic human endeavors of being a self, feeling well, and engaging with others and one's world. Unfortunately, this focus on detailed description did not lend itself to general predictions about how universal psychological models might need to be shaped or reframed by cultural factors, and, for the most part, early cultural and cross-cultural research failed to influence mainstream psychology.

Cultural and cross-cultural research gained focus and predictive power with the field's convergence on a particular pair of cultural constructs, individualism and collectivism.

Correspondence should be addressed to Daphna Oyserman, The University of Michigan, The Institute for Social Research, 426 Thompson Ave., Ann Arbor, MI 48106-1248. E-mail: daphna.oyserman@umich.edu
While the term individualism was not coined by Hofstede (1980), who assessed culture through a workplace survey, his distillation of culture and cross-cultural difference in terms of individualism and three other societal-level culture constructs (power distance, masculinity, and uncertainty avoidance) galvanized the field. By simplifying all of the nuance and complexity of culture into a more straightforward set of basic constructs, Hofstede's (1980) model allowed researchers to begin to ask how cultural factors such as individualism might have shaped psychological theories previously assumed to be universal. Indeed, the follow-up research was almost completely focused on the domain of individualism (later separated into individualism and collectivism).

Broadly speaking, cultural research on individualism can be thought of in terms of a progression. Initial research pulled together many idiosyncratic differences within the individualism rubric and assumed a single scale, with individualism at one end and collectivism at the other. After progress was made with this simple model, a second wave of research included both individualism and collectivism as orthogonal factors. These models contrasted individuals and cultures as either individualistic or collectivistic. The third wave of research conceptualizes individualism and collectivism as more fluid and dynamic features of culture. This third wave of research links cultural research with experimental social psychology methodology by assuming that both individualistic and collectivistic cultural frames are universally available but differentially likely to be brought online into work- 
ing memory. This recent development assumes that cultures differ in the number of situations in which individualism or collectivism are cued, but that both individualism and collectivism are universally cue-able and, once cued, produce the same effects across societies (for a review, see Oyserman, Coon, \& Kemmelmeier, 2002a; Oyserman, Kemmelmeier, \& Coon, 2002b). Using priming techniques, this research has begun to provide insight into how culture matters-influencing what is seen, what is remembered, and how information is processed.

While fruitful, cultural and cross-cultural researchers do not see individualism and collectivism research alone as sufficient in scope to model all-important cultural differences. Indeed, individualism was the second cultural factor Hofstede (1980) identified; power distance was the first. Power distance involves the extent to which a society accepts and views as inevitable or functional human inequality in power, wealth, and prestige (Hofstede, 1980). Individualism highlights human differences, including awareness of unequal distribution of valued characteristics; power distance highlights the extent to which these individual differences are related directly to other inequalities in power, wealth, or prestige, and the extent to which these inequalities are seen as legitimate or illegitimate. By institutionalizing certain links between individual or group characteristics and inequalities in power, power distance as a cultural frame is assumed to provide legitimacy and the sense of fair treatment. That is, power distance has to do with the perceptions and responses of those both high and low in power within a particular system.

Because goals can mostly only be carried out in conjunction and collaboration with others, power, equality, and dependence are fundamental elements of any social structure or culture-yet, the effects of high and low power have not been much studied in the social sciences (see, however, Fiske, 1991; Keltner, Gruenfield, \& Anderson, 2003). Fiske (1991) and Keltner et al. (2003) both present broad reviews arguing that all relationships involve hierarchy, and that power is a pervasive part of human society. Within cultural and cross-cultural psychology, there were a number of attempts to move the field beyond individualism and collectivism and to take up consideration of how authority, power, and hierarchy are integrated into societies. Unfortunately, until now, efforts such as Michael Bond's (1996) work on Chinese culture, Alan Fiske's (1991) taxonomy of basic social relationships, and Triandis' (1995) focus on horizontal/vertical individualism and collectivism have for the most part failed to focus cultural psychology as a whole on the implications of power as a cultural construct.

In their lead article, Shavitt, Lalwani, Zhang, and Torelli (2006) do a service to the field by suggesting a re-emphasis on power and power distance, focusing on Triandis' (1995) horizontal (valuing and emphasizing equality) and vertical (valuing and emphasizing hierarchy) axes. Much like the progression of individualism and collectivism research from a single individualism dimension to a two-construct individualism and collectivism field, Shavitt and her colleagues remind us that research on power has to investigate both power differential (or a vertical focus) and equality (or a horizontal focus), because equality is not the same as accepting or not accepting a power differential. As described in the lead article, the horizontal/vertical (HV) description is embedded within the description of individualism and collectivism focusing on assessment of horizontal individualism (HI), vertical individualism (VI), horizontal collectivism (HC), and vertical collectivism (VC). Shavitt and colleagues show evidence that the HI-HC-VI-VC method of integrating the study of power and equality with the study of individualism and collectivism captures previously unexplored differences, for example, in social desirability responding.

However, by considering power as an horizontal/vertical axis within the individualism-collectivism framework, it is not possible to disentangle effects of power or equality from effects of individualism or collectivism. As Shavitt and colleagues note, collectivism is correlated robustly with verticality. Moreover, the current operationalization of verticality (acceptance of power distance) and horizontality (equality) does not distinguish between having and not having power. Recent programs of research suggest that what matters is not simply whether a power differential existswhat matters is having or not having power (see Fiske, 2001; Keltner et al., 2003). It may therefore be more advantageous to treat high power, low power, and equality as separate aspects to allow for more synergy between cross-cultural psychology and an already existing field of research on the effects of having versus not having power in situations that are characterized by a power differential, and of being equal in equality situations.

\section{INDIVIDUALISM AND COLLECTIVISM, HIGH POWER, LOW POWER, AND EQUALITY}

\section{Assessment and Conceptualization}

Hofstede (1980) described individualism and not individualism and collectivism; that is, he made the assumption that the opposite of individualism is collectivism, and that once high and low individualism were operationalized, there would be no need to separately operationalize collectivism. This simplifying assumption was accepted by many researchers, but a recent meta- analysis (Oyserman et al., 2002a) showed that individualism and collectivism are orthogonal. This means that individualism and collectivism, and their effects on psychological processes, must each be tested separately-presumably because both exist to some extent in all societies and influence psychological processes when they are made salient or brought to mind via situational priming. This same argument could be made for high power, low power, and equality. It seems reasonable to assume two orthogonal axes 
of power (high and low), and equality (high and low), where low power may assume the form of dependence.

Implications of Individualism and Collectivism, High Power, Low Power, and Equality

To the extent that groups differ in individualism and collectivism, and European Americans (and Western Europeans) are high in individualism, this implies that the psychological models developed within this cultural frame-of self-concept content and functioning, well-being, attribution style, and relationality - are not universal models but simply models derived from and applicable to an individualistic worldview, and that other models must be developed for other groups (see Markus \& Kitayama, 1991; Oyserman et al., 2002a; Oyserman et al., 2002b). A case can be made that contemporary American psychological research is particularly suited to an individualistic worldview as demonstrated by research on social and nonsocial cognition, including research on self-concept and relationality. Difference in power distance or horizontal/vertical individualism within Europe are noted in Shavitt and colleagues' lead article (2006) and conceptual reviews focused on the consequences of power differential on self-concept, interpersonal relations, and cognition (e.g., Fiske, 2001; Keltner et al., 2002) suggest that American society includes both. In the next section, I list some implications of these differences in cultural frames.

With regard to self-concept, individualism implies that creating and maintaining a positive sense of self is a basic human endeavor that involves feeling good about oneself and defining oneself in terms of differentiable attitudes, traits, and opinions. Collectivism, on the other hand, implies that being in a group is a basic human endeavor, so that self-concept involves group membership and self-defining traits should reflect the goals of collectivism, such as sacrifice for the common good and maintaining harmonious relationships with close others. These images are not contradictory and together describe the basic self-concept motives described in the literature-learning about, feeling good about, and improving oneself, and using self-knowledge to navigate the social world (e.g., Oyserman, 2001). However, fitting the implied dominance of individualism, self-esteem research and theorizing has long dominated the field (Baumeister, 1998). Broadening the focus beyond individualistic assumptions should facilitate attention to the other self-goals. Examination of high power, low power, and equality as cultural dimensions may facilitate linkage of self-concept literature to research on social identities, stereotyping, and stigma that suggest that how the self is conceptualized is influenced by the relative power of the in-group to control access to valued resources.

With regard to interpersonal and intergroup relations, individualism implies impermanence-one can become estranged from one's family, immigrate to a new country, convert to a new religion, and lose touch with friends. Collectivism implies permanence-one can never really leave these groups. Groups and boundaries are important and fixed facts of life to which one must accommodate, so that indirect communication and methods are prized as ways to avoid disrupting in-group harmony. While individualism and collectivism research has provided insight into cross-cultural differences in use of equity, equality, and generosity norms (for a review, see Oyserman et al., 2002a), it is entirely possible that this work has conflated individualism and collectivism with high power, low power, and equality axes of culture. In part, that is because researchers often neglected to assess the cultural construct they believed to be at the root of the cross-national differences they found, and simply asserted that they are due to individualism and collectivism. Fiske (1991) suggested that all societies have some access to relationships that are equitable as well as those that are based in differential power-although societies are likely to differ in the proportion of such relationships they are likely to experience in their daily lives. For example, ease of interaction with strangers and obligation to in-groups may be results of individualism and collectivism, or they may be due to equality and power-dependence. As Shavitt and colleagues note in their discussion of verticality, power differential may often be positively associated with collectivism. This likely positive association further complicates our ability to disentangle the constructs unless they are separately experimentally manipulated. Moreover, disentangling and separately manipulating the power and equality constructs will allow for progress integrating related cultural factors such as honor, which are likely to be separate from individualism and collectivism yet carry power-related meaning.

With regard to cognition, individualism implies that judgment, reasoning, and causal inference are generally oriented toward the person rather than the situation or social context, because the decontextualized self is assumed to be a stable causal nexus. Consequently, individualism promotes a decontextualized reasoning style that assumes that social information is not bound to social context. Oyserman and colleagues have described this style as a 'separate-andpull-apart' style as distinct from a situation-specific relational 'embed-and-connect' style (Markus \& Oyserman, 1989; Oyserman et al., 2002b; Oyserman \& Lee, in press).

Follow-up research documents that priming individualism and collectivism shifts processing among Western (European Americans; Kühnen \& Oyserman, 2002; Haberstroh, Oyserman, Schwarz, Kühnen, \& Ji, 2002), and Eastern (Korean; Oyserman, Sorensen, Cha, \& Schwarz, 2006) participants. The differences observed in priming experiments are compatible with the differences observed in cross-national comparisons between the United States and China (Haberstroh et al., 2002; Nisbett, 2003) and the United States and Japan (Kitayama, Duffy, Kawamura, \& Larsen, 2003). Primed or chronically collectivism-focused participants process for contextual, relative information; primed or chronically individualism-focused participants process for main points or figures-not background. 
Research on cognitive style has utilized the full range of procedures (priming, assessment of individualism and collectivism, and cross-national comparison) and, in this sense, may be more robustly attributable to individualism and collectivism than is the case for research on interpersonal and intergroup relations. However, there is no reason to assume that the only cross- cultural differences in cognitive style are those "pull-apart" and "connect" procedural operating schemas attributable to individualism and collectivism. Another important and relevant distinction is global versus local processing. Social cognition research suggests that individuals generally process information at a global, schema-driven level and utilize a more specific, detail-oriented approach only under certain circumstances (Fiske \& Taylor, 1991).

Asking how power influences social judgment, Fiske (2001) conducted a series of studies demonstrating that processing of information differs across situations in which power is equal, power is unequal and one has it, and power is unequal and one does not have it. In each case, power is primed via participation in tasks in which one's outcomes are or are not mutually dependent upon another's efforts. When both actors are dependent on each other for their outcomesa situation of equality - they are particularized in their processing of the other's strengths and weaknesses. When one actor depends on the other but the dependence is not mutual, processing style shifts to a more schema-driven global processing for those with power, and to detail-focused attention with a tendency to create a positive synthesis for those without power.

While Fiske (2001) does not use these terms, this research suggest that power-dependence and equality are likely to relate to the tendency to use global as compared to local processing styles. Global processing-described as seeing the forest but not the trees-emphasizes the whole gestalt and seems to be the prerogative of those who have power, not those who lack power. Local processing is used both by those who are in a situation of equality, as well as those who are in a situation in which they lack power. In addition to research on power, global versus local processing style has been studied as it relates to mood (Gasper \& Clore, 2002) and self-regulatory focus (Föster \& Higgins, 2005). Bad mood and a caution-oriented focus on fulfilling duties and obligations have both been associated with local processing, while good mood and a promotion-oriented focus on attaining success have both been associated with global processing via experimental manipulation. Throughout, these influences are consistent with the assumption that processing strategies are situated and tuned to meet current situational requirements (for a review, see Schwarz, 2002). In the case of power, when the situation evokes high power, a global, schema-, stereotype-, or expectancy-driven style is sufficient; when the situations evokes low power or equality, a local, detail-oriented style is necessary.

Power itself has been related to both mood and selfregulatory focus (for a review, see Keltner et al., 2003).
Global and local styles are similar to, but not identical with, the cognitive styles previously related to individualism and collectivism, and can contribute to a fuller account of culturally embedded cognitive styles. Local processing is not the same as the kind of context-dependent and relational (Oyserman et al., 2002b) or holistic (Nisbett, 2003) processing attributed to collectivism in general, or to Chinese culture in particular. Local processing is primed by situations of equality, as well as by situations of dependence and lack of power. Global processing is similar to, but not the same as, the pull-apart (Oyserman et al., 2002b; Oyserman \& Lee, in press) or analytic processing (Nisbett, 2003) attributed to individualism, generally, or Western culture, specifically. Global processing is primed by situations of power distance in which one has power.

This brief overview suggests that it will be advantageous to isolate the role of high power, low power (dependence), and equality, and to develop experimental procedures that allow tests of causality. Correlational research on individualism and collectivism-showing an association with content of self-concept, interactional style, and cognitive processes - was strengthened and moved forward by shifting to priming techniques, and the same may be expected for high power, low power, and equality. These constructs are both universal-all humans are sensitive to cues about power and respond differently when they do or do not have power-and likely to differ cross-culturally in the extent to which they are chronically accessible. Cues about power and equality are likely to influence everything from overt behavior to strategies of information processing.

Thus, thinking about high power, low power, and equality as separate constructs suggests, first, that currently used experimental procedures for priming individualism and collectivism should be tested to see if they carry with them activation of power and equality constructs. This could be done using simple techniques such as word completion tasks or semantic priming tasks, to see if words related to power and equality are more or less accessible after individualism and collectivism priming. Second, thinking about power and equality as separate constructs also suggests that their influence can and should be tested via standard priming procedures (see also Meyers-Levy, 2006). One promising task involves having participants fill out relevant rating scales (e.g., a social hierarchy attitudes scale) before rather than after the dependent variable is assessed. Alternatively, participants could be primed with high or low power or equality concepts through a scrambled sentence task (e.g., Smith \& Trope, 2006), through exposure to images of power and hierarchy versus equality, or through relevant scenarios.

Just as the field of cultural psychology has moved beyond the initial assumption that cultures are high either in individualism or collectivism, power and equality constructs are also likely to be culturally universal. Equality relationships and power-differential relationships are basic to the social units that make up societies: family, school, or work; one's 
peer and sibling relationships; and interactions between racial, ethnic, and religious groups. Although societies may be differentially likely to make having high or low power or being equal salient, these constructs should have similar effects across societies once they are brought to mind.

Because high power, low power (dependence), and equality are not narrowly defined, priming power is likely to influence each of the domains previously studied in individualism and collectivism research. In terms of self-concept, priming power, equality, and dependence is likely to make salient different ways of defining the self. In terms of interpersonal and intergroup relationships, priming power, equality, and dependence are likely to make salient different ways of making sense of, and engaging with, others. Yet, it is in the domain of cognition, memory, and perception for which research on power may provide the most promising venues for new research. As Shavitt and colleagues note, taking power into account is not redundant with taking individualism and collectivism into account. Understanding high power, low power (dependence), and equality is a promising next step in cultural and cross-cultural research.

\section{ACKNOWLEDGMENTS}

Thanks to Johannes Keller, Ayse Uskul, Nicholas Sorensen, and Mesmin Destin for their comments.

\section{REFERENCES}

Baumeister, R. (1998). The self. In D. Gilbert, S. Fiske, \& G. Lindzey (Eds.), Handbook of social psychology (Vol. 1, pp. 680-740). New York: Oxford University Press.

Bond, M. (1996). Chinese values. In M. Bond (Ed.), The handbook of chinese psychology (pp. 208-226). Hong Kong: Oxford University Press.

Fiske, A (1991). Structures of social life: The four elementary forms of human relations. New York: Free Press.

Fiske, S. (2001). Effects of power on bias: Power explains and maintains individual, group, and societal disparities. In A. Lee-Chai \& J. Bargh (Eds.), The use and abuse of power: Multiple perspectives on the causes of corruption (pp. 181-194). Philadelphia, PA: Psychology Press.

Fiske, S., \& Taylor, S. (1991). Social cognition. New York: McGraw-Hill.

Föster, J., \& Higgins, T. (2005). How global versus local perception fits regulatory focus. Psychological Science, 16, 631-636.
Gasper, K., \& Clore, G. (2002). Attending to the big picture: Mood and global versus local processing of visual information. Psychological Science, $13,34-40$.

Haberstroh, S., Oyserman, D., Schwarz, N., Kühnen, U., \& Ji, L-J. (2002). Is the interdependent self more sensitive to question context than the independent self? Self-construal and the observation of conversational norms. Journal of Experimental Social Psychology, 38, 323-329.

Hofstede, G. (1980). Culture's consequences. New York: Sage.

Keltner, D., Gruenfield, J., \& Anderson, C. (2003). Power, approach, and inhibition. Psychological Review, 110, 265-284.

Kitayama, S., Duffy, S., Kawamura, T., \& Larsen, J. (2003). Perceiving an object and its context in different cultures. Psychological Science, 14, 201-206.

Kühnen, U., \& Oyserman, D. (2002) Thinking about the self influences thinking in general: Cognitive consequences of salient self-concept. Journal of Experimental Social Psychology, 38, 492-499.

Markus, H., \& Kitayama, S. (1991). Culture and the self: Implications for cognition, emotion, and motivation. Psychological Review, 20, 568-579.

Markus, H., \& Oyserman, D. (1989). Gender and thought: The role of the self-concept. In M. Crawford \& M. Gentry (Eds.), Gender and thought: Psychological perspectives (pp. 100-127). New York: Springer-Verlag.

Meyers-Levy, J. (2006). Using the horizontal/vertical distinction to advance insights into consumer psychology. Journal of Consumer Psychology, 16(4), 347-352.

Nisbett, R. (2003). The geography of thought: How Asians and Westerners think differently ... and why. New York: Free Press.

Oyserman, D. (2001). Self-concept and identity. In A. Tesser \& N. Schwarz (Eds.), Blackwell handbook of social psychology (pp. 499-517). Malden, MA: Blackwell.

Oyserman, D., Coon, H., \& Kemmelmeier, M. (2002a). Rethinking individualism and collectivism: Evaluation of theoretical assumptions and meta-analyses. Psychological Bulletin, 128, 3-73.

Oyserman, D., Kemmelmeier, M., \& Coon, H. (2002b). Cultural psychology, a new look. Psychological Bulletin, 128, 110-117.

Oyserman, D., \& Lee, S. (in press). Priming "culture": Culture as situated cognition. In S. Kitayama and D. Cohen (Eds.), Handbook of Cultural Psychology. New York: Guilford Press.

Oyserman, D., Sorensen, N., Cha, O., \& Schwarz, S. (2006). Thinking about "me" or "us" in East and West: Priming independence and interdependence and Stroop performance. Manuscript under editorial review.

Schwarz, N. (2002). Situated cognition and the wisdom of feelings: Cognitive tuning. In L. Feldman Barrett \& P. Salovey (Eds.), The wisdom in feelings (pp.144-166). New York: Guilford.

Shavitt, S. Lalwani, A. K., Zhang, J., \& Torelli, C. J. (2006). The horizontal/ vertical distinction in cross-cultural consumer research. Journal of Consumer Research, 16(4), 325-342.

Smith, P. K., \& Trope, Y. (2006). You focus on the forest when you're in charge of the trees: Power and abstract information processing. Journal of Personality and Social Psychology, 90, 578--596.

Triandis, H. C. (1995). Individualism and collectivism. Boulder, CO: Westview. 